\title{
Berberine reduce allergic inflammation in a house dust mite allergic rhinitis mouse model*
}

\author{
Boo-Young Kim', Hyang Rim Park', Hyeon Gun Jeong², Soo Whan Kim¹ \\ 'Department of Otolaryngology-Head and Neck Surgery, The Catholic University of Korea, College of Medicine, Seoul, Korea \\ ${ }^{2}$ Ahngook Pharm. RAD center, Korea
}

Rhinology 53: 353-358, 2015

DOI:10.4193/Rhino15.028

*Received for publication:

February 5, 2015

Accepted: April 29, 2015

\begin{abstract}
Background: Berberine (Ber), used widely as an antibacterial, antifungal, and anti-inflammatory drug, has long been used as a gastrointestinal remedy in Chinese traditional medicine. Recent reports have suggested that Ber suppresses Th17 responses that was mediated by direct actions on T cells and thymic stromal lymphopoietin production in primary mast cells. It has been suggested that Ber may be useful in treating allergic response. The purpose of this study was to assess the effects of Ber treatment on allergic inflammation in an allergic rhinitis mouse model and to examine the underlying mechanism(s).
\end{abstract}

Methods: BALB/c mice were divided into control, Derf with no treated (Derf), Ber treated, and Ber with anti-C25 monoclonal antibody treated (Ber + anti-CD25) groups. All mice, with the exception of the control group, were sensitized with an intraperitoneal i.p. injection of Dermatophagoides farinae (Derf). Mice in the Ber and Ber + anti-CD25 group were treated intranasally with $10 \mu \mathrm{g} / \mathrm{mL}$. Then, 1 week after sensitization, all mice were challenged intranasally with $20 \mu \mathrm{g}$ Derf for 5 consecutive days. Mice in the anti-CD25 group were treated intraperitoneally with $250 \mu \mathrm{g}$ anti-CD25 monoclonal antibody 1 day before the first intra-nasal challenge with Derf. Allergic symptom scores, eosinophil counts, and serum Derf-specific lgE levels were measured. T-bet, GATA-3, interferon- $\gamma$ (IFN- $\gamma$ ), interleukin (IL)-10, IL-13, and Foxp3 expression was examined by real-time polymerase chain reaction and Western blotting. $\mathrm{CD} 4{ }^{+} \mathrm{CD} 25^{+}$Foxp3 ${ }^{+} \mathrm{T}$ cells were assessed by flow cytometry.

Results: Symptom scores, serum Derf-specific IgE levels, GATA-3 mRNA levels, T-bet mRNA levels, and tissue eosinophil counts were decreased in the Ber versus the Derf group. In the Ber + anti-CD25 group, serum IL-10 levels were decreased versus the control, Derf, and Ber groups. In the Ber + anti-CD25 mAb groups, Foxp3 mRNA levels were decreased versus the control group. In the Ber group, Foxp3 mRNA levels were increased versus the control group. In the Ber group, the percentage of CD4 $4^{+} \mathrm{CD} 25^{+} \mathrm{Foxp} 3^{+}$ $T$ cells was increased versus the Derf group. The percentage of $C D 4^{+} C D 25^{+}$Foxp $3^{+} T$ cells was increased in the Ber versus the Derf groups.

Conclusions: In our study, Ber reduced allergic inflammation significantly. Moreover, our findings suggest that the mechanism of action of Ber may be via $\mathrm{CD} 4^{+} \mathrm{CD} 25^{+} \mathrm{Foxp}^{+}$Treg cells, possibly through not only by increasing their numbers but also altering their function.

Key words: Berberine, allergic rhinitis, regulatory T cells, a mouse model, house dust mite, anti-CD25 monoclonal antibody, CD4 ${ }^{+}$CD25 ${ }^{+}$Foxp $^{+}$T cells

\section{Introduction}

Allergic rhinitis (AR) affects $\sim 20 \%$ of people of all ages. The risk of developing $A R$ is considerably higher in people with asthma or eczema ${ }^{(1)}$. Unfortunately, the symptoms of AR cannot usually be controlled completely with any combination of present environmental measures, medications, or immunotherapy. 
Berberine (Ber), an isoquinoline-derivative alkaloid, is a major component of Rhizoma coptidis (Huanglian), a traditional Chinese medicine. Many studies have demonstrated that Ber has many pharmacological actions, including anti-inflammatory, immunostimulatory, and antidiarrheal activities. Recent reports have suggested that Ber suppresses inflammation in collagen-induced arthritis, an animal model of human rheumatoid arthritis, experimental type I diabetes, and experimental autoimmune encephalomyelitis, an animal model of human multiple sclerosis ${ }^{(2-5)}$. There have been reports that the inhibitory effect of Ber on Th17 responses was mediated by direct actions on T cells as well as indirect effects, via dendritic cells, in autoimmune diseases ${ }^{(6)}$. In other studies, Ber inhibited thymic stromal lymphopoietin (TSLP) production in primary mast cells. Thus, it has been suggested that Ber may be useful in treating inflammation and atopic dermatitis (7). However, limited data are available regarding the anti-allergic effects of Ber in an AR model. The purpose of this study was to investigate the effects of Ber in a mouse model of AR and to examine the underlying mechanism(s) using various cytokine and anti-CD25 monoclonal antibody studies.

\section{Materials and methods}

Experimental animals

Six-week-old healthy female BALB/c mice (20 - $30 \mathrm{~g})$ were used in the present study. This study was performed with the approval of the Institutional Animal Care and Use Committee at the Catholic University of Korea.

\section{Reagents}

Dermatophagoides farinae (Derf) crude body extract (Arthropods of Medical Importance Resource Bank, College of Medicine, Yonsei University, Seoul, Korea) was used as the allergen. The Derf crude extract was solubilized and stored at $70^{\circ} \mathrm{C}$ and dissolved prior to use. The anti-Ber (Sigma-Aldrich, St. Louis, MO, USA) and anti-CD25 (clone PC61, eBioscience, San Diego, CA, USA) monoclonal antibodies were prepared in sterile phosphate-buffered saline (PBS).

\section{Production of the AR model and treatment protocol}

Forty mice were randomized into one of four groups: control (n $=10)$, Derf (AR, $n=10), \operatorname{Ber}(n=10)$, and Ber + anti-CD25 mAb $(n=10)$. Allergen sensitization and challenge for development of the AR murine model are summarized in Figure 1. Briefly, on days 0,7 , and 14 , all mice, except those in the control group, were immunized by intraperitoneal injection of $100 \mu \mathrm{g}$ Derf and $1 \mathrm{mg}$ aluminum hydroxide (Sigma-Aldrich). Mice in the Ber and Ber + anti-CD25 group were treated intranasally with $10 \mu \mathrm{g} / \mathrm{mL}$ Ber on days 21, 22, 23, 24, and 25. Then, 1 week after sensitization, all mice were challenged intranasally with $20 \mu \mathrm{g}$ Derf for 5 consecutive days. Mice in the anti-CD25 group were treated intraperitoneally with $250 \mu \mathrm{g}$ anti-CD25 monoclonal antibody 1 day before the first intra-nasal challenge with Derf (on day 20). The control group received PBS intranasally instead of Derf ${ }^{(8)}$.

Evaluation of allergic symptoms induced after allergen challenge

The numbers of sneezing and nose-rubbing motions during the 15-min period after the final allergen challenge were recorded and compared among the experimental groups by observers blinded to the experimental groups ${ }^{(9)}$.

\section{Nasal mucosal tissue evaluation}

Mice were sacrificed and decapitated $24 \mathrm{~h}$ after the final allergen challenge. The heads were fixed in $4 \%$ paraformaldehyde for 3 days at $4^{\circ} \mathrm{C}$, washed in running water, decalcified for 3 days with Calci-Clear Rapid (National Diagnostics, Atlanta, GA, USA)10 at room temperature, dehydrated by passage through a graded alcohol series, and embedded in paraffin blocks. The blocks were cut into 4- $\mu \mathrm{m}$ sections and stained with hematoxylin and eosin to evaluate the general morphology and the number of eosinophils in the lamina propria of nasal mucosa. Eosinophils were counted under a light microscope. The average number of cells was counted in four areas around the nasal septa. The individual who counted the eosinophils was blinded to the animals' group assignments.

\section{Derf-specific immunoglobulin E levels in serum}

Serum Derf-specific immunoglobulin E (IgE) levels were measured using an ELISA kit (Bethy, Montgomery, TX, USA).

\section{Real-time polymerase chain reaction (PCR)}

The nasal mucosa was removed for detection of IFN- $\gamma$, T-bet, GATA-3, IL-4, IL-13, IL-10, and Foxp3 mRNA using real-time PCR. Total RNA was extracted from nasal mucosa using the TRIzol reagent (Invitrogen, Carlsbad, CA, USA), and the first strand was reverse-transcribed using random primers (TaKaRa, Otsu, Japan) ${ }^{(11,12)}$. The oligonucleotide primer sequences were as follows: IFN- $\gamma$ forward primer, 5' AGAGCCAGATTATCTCTTTCTACCTCAG-3' and IFN- $\gamma$ reverse primer, $5^{\prime}$ CCTTTTTCGCCTTGCTGTTG-3'; T-bet forward primer, 5' GCCAGGGAACCGCTTATA-3' and T-bet reverse primer, 5' CCTTGTTGTTGGTGAGCTTTA-3'; GATA-3 forward primer, 5' CTGGATGGCGGCAAAGC-3' and GATA-3 reverse primer, 5' GTGGGCGGGAAGGTGAA-3'; IL-4 forward primer, 5' TCAACCCCCAGCTAGTTGTC-3' and IL-4 reverse primer, 5' AAATATGCGAAGCACCTTGG-3'; IL-13 forward primer, 5' CCTCTGACCCTTAAGGAGCTTAT-3' and IL-13 reverse primer, 5' CGTTGCACAGGGGAGTCT-3-; Foxp3 forward primer, 5' GAAAGCGGATACCAAATGA-3' and Foxp3 reverse primer, 5' CTGTGAGGACTACCGAGCC-3'; IL-10 forward primer, 5' GCTCTTACTGACTGGCATGAG-3' and IL-10 reverse primer, 5' CGCAGCTCTAGGAGCATGTG-3'; Foxp3 forward primer, 5' 

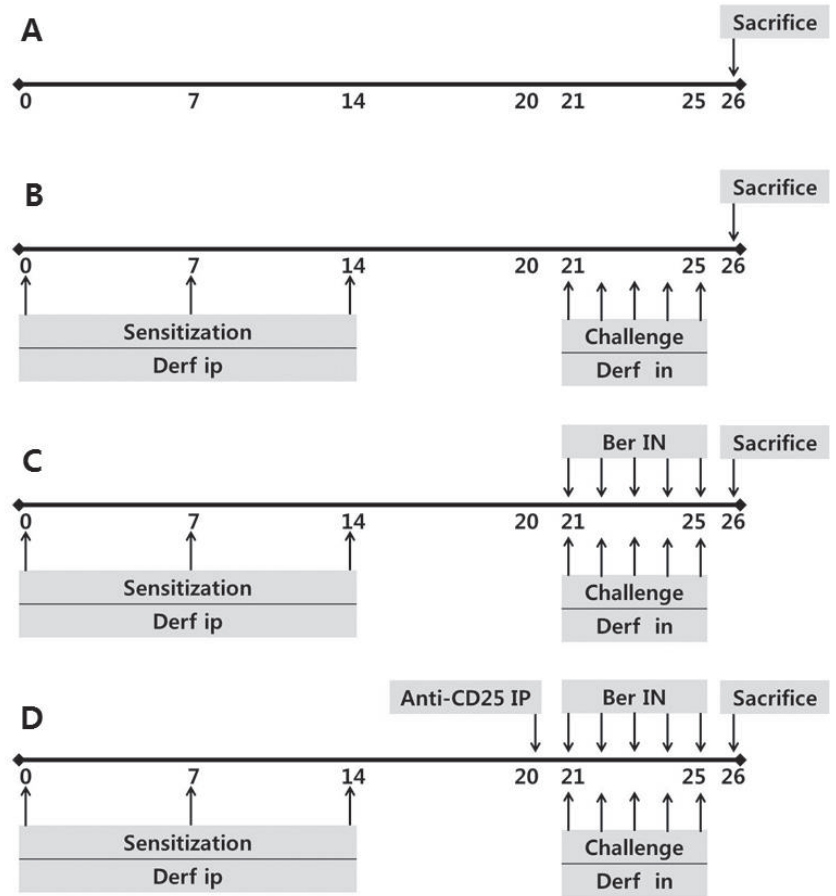

Figure 1. Schematic representation of the experimental allergic rhinitis model and treatment protocol. Control (A), Derf with no treated (B), Ber treated (C), and Ber with anti-C25 monoclonal antibody treated groups (D). Ber, Berberine; anti-CD25, anti-CD25 monoclonal antibody; Derf, Dermatophagoides farina; IP (ip), intraperitoneal administration; IN (in), intranasal administration. Data are expressed as days.
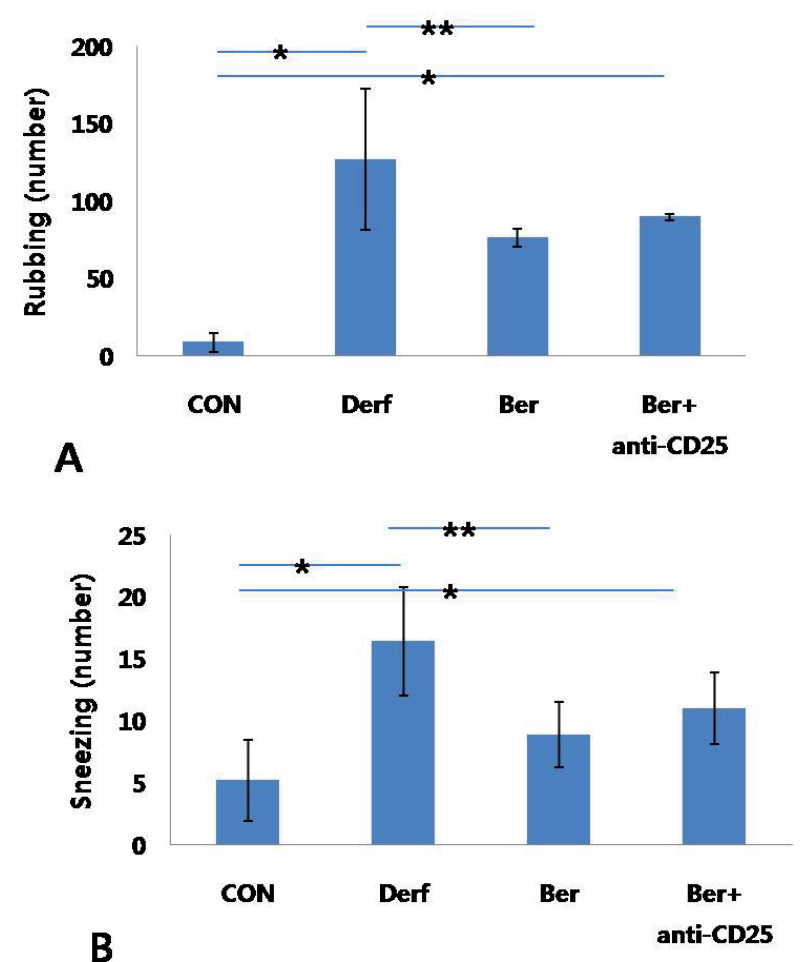

Figure 2. Nasal symptom score. Rubbing (A) and sneezing (B). Error bars indicate standard deviations. ${ }^{*} \mathrm{p}<0.05$, vs. the Derf group, ${ }^{* *} \mathrm{p}<0.05$, vs. the control group.
GAAAGCGGATACCAAATGA-3' and Foxp3 reverse primer, 5' CTGTGAGGACTACCGAGCC-3'; glyceraldehyde 3-phosphate dehydrogenase (GAPDH) forward primer, 5'-GCACAGTCAAGGCCGAGAAT-3' and GAPDH reverse primer, $5^{\prime}$-GCCTTCTCCATGGTGGTGAA-3'. The levels of IFN- $\gamma$, T-bet, GATA-3, IL-4, IL-13, IL-10, Foxp3, and GAPDH mRNA expression were determined by real-time PCR using the CFX96 Real Time PCR Machine (Bio-Rad, Hercules, CA, USA) and iQ SYBR Green Supermix (Bio Rad). The results were normalized relative to GAPDH expression and are shown as the fold increases over the expression of the control group.

\section{Western blot analysis}

Western blot analysis was used to determine GATA-3, IL-13, IL-10, and Foxp3 protein levels within the nasal mucosa from the control, Derf, Ber and Ber + anti-CD25 groups. Results were based on the gray-scale ratios between the target gene and GAPDH to indicate the relative levels of the proteins encoded by the target genes using anti-GATA-3 (Santa Cruz Biotechnology, Santa Cruz, CA, USA), -IL-13, -IL-10, and -Foxp3 (eBioscience, San Diego, CA, USA) antibodies.

\section{Flow cytometry}

For cell-surface staining, aliquots of $10^{6}$ splenic mononuclear cells were incubated with fluorescein isothiocyanate (FITC)conjugated anti-mouse CD4 (GK1.5) antibody (eBioscience, San Diego, CA, USA). For intracellular staining, cells stained with CD4 were incubated with fixation/permeabilization working solution, and Fc receptors were blocked with excess mouse Fc block. Cells were then stained with anti-phycoerythrin (PE)-Cy5-conjugated anti-mouse Foxp3 (FJK-16s) and anti-allophycocyanin (APC)CD25 antibodies (eBioscience). $C D 4{ }^{+} C D 25^{+}{ }^{+}{ }$oxp $3^{+} T$ cells were analyzed by flow cytometry (FACSCalibur; Becton Dickinson, San Jose, CA, USA)(12,13).

\section{Statistical analysis}

All measured parameters are expressed as means \pm standard deviation. Differences among groups were analyzed using the Kruskal Wallis one-way ANOVA test. In cases of statistical significance, the ranked parameters were compared by one-way analysis of variance and Bonferroni's multiple comparison tests (PASW Statistics 18; SPSS Inc., Chicago, IL, USA). In all analyses, $p$ values $<0.05$ were considered to indicate statistical significance. 


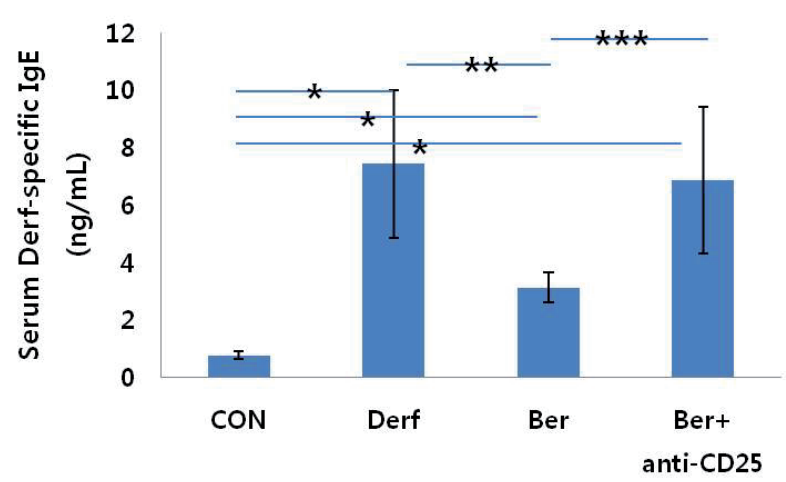

Figure 3. Serum Derf-specific immunoglobulin E. Error bars indicate standard deviations. ${ }^{*} p<0.05$, vs. the Derf group, ${ }^{* *} p<0.05$, vs. the control group. ${ }^{* * *} \mathrm{p}<0.05$, vs. the Ber with anti-C25 monoclonal antibody treated (Ber+anti-CD25) group.

\section{Results}

\section{Allergic symptoms}

Allergic symptoms were determined by the numbers of nasal rubbing motions and sneezes during the 15 -min period after the final challenge. The numbers of nasal rubbing motions were $8.6 \pm 6.26$ in the control group, $126.83 \pm 45.6$ in the Derf group, $7.6 \pm 5.65$ in the Ber group, and $89.5 \pm 2.12$ in the Ber + anti-CD25 group. The numbers of sneezes were $5.2 \pm 3.27$ in the control group, $16.37 \pm 4.37$ in the Derf group, $8.83 \pm 2.63$ in the Ber group, and $11 \pm 2.91$ in the Ber + anti-CD25 group. The number of nasal rubbing motions was significantly higher in the Derf group than in the control or Ber groups (control, $p<0.001$; Ber, $p=0.013$; Figure 2A). The number of sneezes was significantly higher in the Derf group than in the control or Ber groups (control, $\mathrm{p}<0.001$; Ber, $\mathrm{p}=0.005$; Figure 2B).

\section{Serum Derf-specific IgE}

Serum Derf-specific IgE levels were significantly higher in the Derf group $(7.43 \pm 2.56 \mathrm{ng} / \mathrm{mL})$ than in the control $(0.77 \pm 0.14$ $\mathrm{ng} / \mathrm{mL}, \mathrm{p}=0.013)$ and $\operatorname{Ber}(3.13 \pm 0.52 \mathrm{ng} / \mathrm{mL}, \mathrm{p}=0.001)$ groups. The Ber group had a significantly lower serum Derf-specific lgE level than the Ber + anti-CD25 group ( $p=0.002$; Figure 3$)$.

\section{Eosinophil counts in the lamina propria}

Figure 4A-D shows eosinophil infiltration in the lamina propria. Eosinophil numbers were $26 \pm 5.65$ in the control group, $92.5 \pm$ 6.36 in the Derf group, $26 \pm 1.73$ in the Ber group, and $78 \pm 4.24$ in the Ber + anti-CD25 group. The eosinophil count was significantly higher in the Derf group than in the control $(p=0.006)$ or Ber $(p=0.029)$ groups (Figure 4E).

\section{Real-time PCR and Western blot analyses}

IFN- $\gamma$ mRNA expression level was significantly higher in the Ber + anti-CD25 group than in the control group $(p=0.045$; Figure
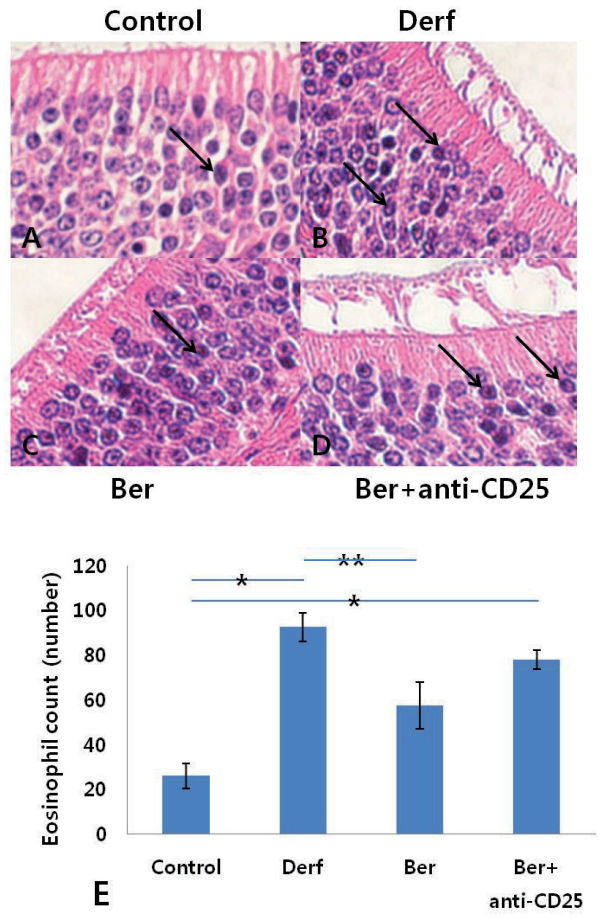

Figure 4. Infiltration of eosinophils (arrows) in the nasal mucosa of BALB/c mice: (A) control group, (B) Derf group, (C) Ber group, and (D) Ber + anti-CD25 group (E) Eosinophil counts in the nasal mucosa of each study group. Error bars indicate standard deviations. ${ }^{*} p<0.05$, vs. the Derf group, ${ }^{* *} p<0.05$, vs. the control group (hematoxylin and eosin staining; original magnification, $\times 400)$.

5A). The T-bet mRNA expression level was significantly higher in the Derf group than in the Ber group ( $p=0.013$; Figure 5B).

GATA-3 mRNA and protein levels were significantly higher in the Derf group than in the control $(p=0.015)$ or Ber groups $(p=$ 0.008). The Ber group had a significantly lower GATA3 mRNA level than did the Ber + anti-CD25 group $(p=0.047$; Figure $5 C, H)$. The IL-4 mRNA level was significantly higher in the Derf group than in the control group $(p<0.002)$ or the Ber group $(p=0.001$; Figure 5D). IL-13 mRNA and protein levels were significantly higher in the Derf than in the control group $(p=0.016)$ and than in the Ber + anti-CD25 group $(p=0.008)$. The Ber group had a significantly lower IL-13 mRNA level than the anti-CD25 group $(p=0.001$; Figure $5 E, H)$. The IL-10 mRNA level and protein levels were significantly higher in the Ber group than in the Ber + anti-CD25 group ( $p=0.003$; Figure 5F, H). The Foxp3 mRNA level and protein levels were significantly higher in the Ber group ( $p$ $=0.001)$ than in the control group $(p=0.001)$ or Derf group $(p=$ 0.023; Figure 5G, H).

Flow cytometric analysis of $\mathrm{CD}^{+} \mathrm{CD}^{+} 5^{+} \mathrm{Foxp}^{+} \mathrm{T}$ cells from splenic mononuclear cells

Cells were sorted based on Foxp3 and CD25 expression levels 

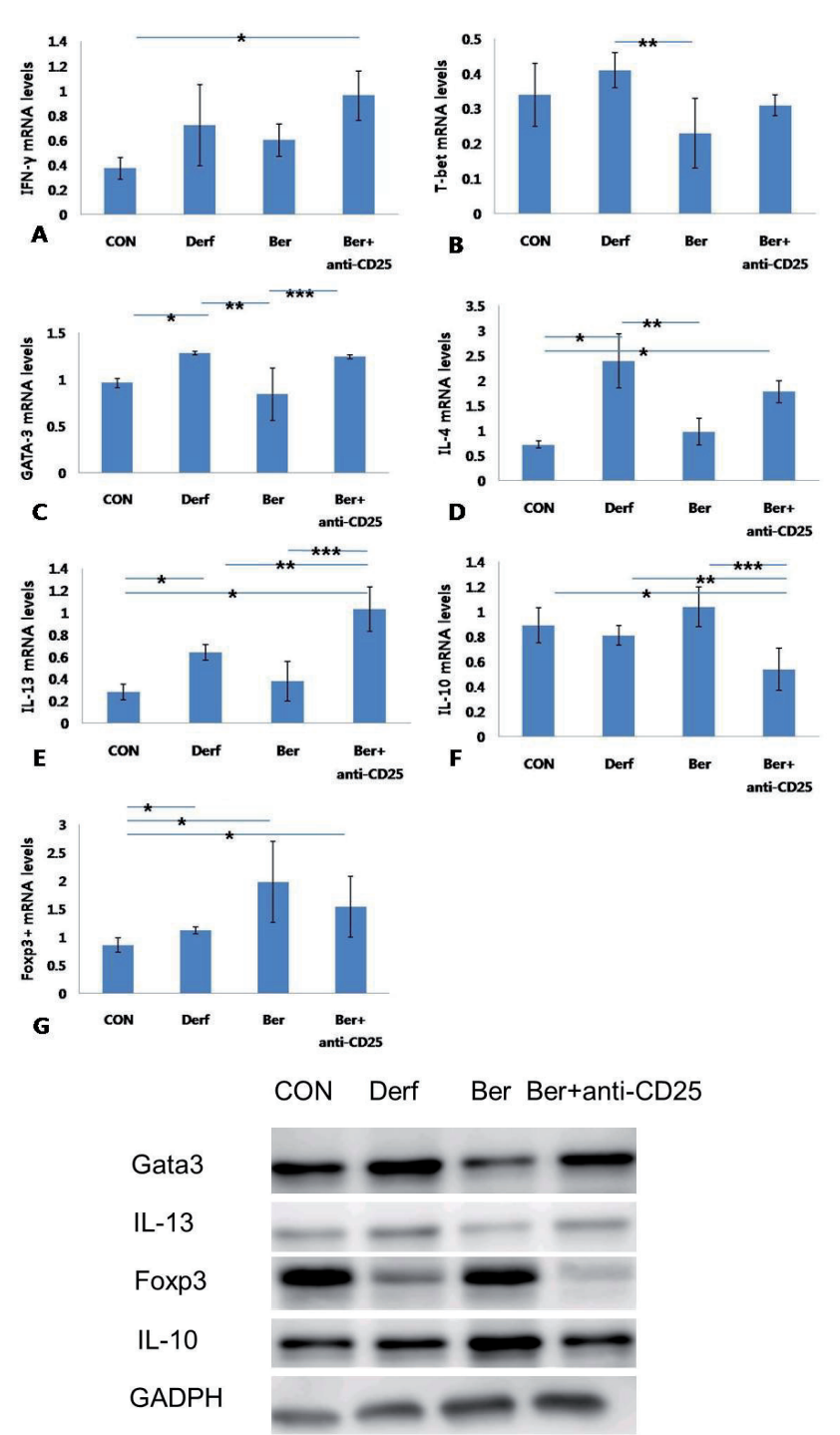

Figure 5. Quantitative analysis of IFN- $\gamma(A)$, T-bet (B), GATA-3 (C), IL-4 (D), IL-13 (E), IL-10 (F) and Foxp3 (G) mRNA expression in nasal mucosa by real-time PCR. The results were normalized relative to GAPDH expression. Error bars indicate standard deviations. ${ }^{*} p<0.05$, vs. the Derf group, ${ }^{* *} \mathrm{p}<0.05$, vs. the control group. $(\mathrm{H})$ Western blot analysis was used to determine GATA-3, IL-13, Foxp3, and IL-10 protein levels.

and whether they expressed CD4 (Figure 6A). CD4 ${ }^{+} \mathrm{CD} 25^{+} \mathrm{Foxp}^{+}$ $T$ cells accounted for $1.125 \pm 0.077 \%$ of all splenic mononuclear cells in the control group, $0.46 \pm 0.1 \%$ in the Derf group, 1.361 $\pm 0.194 \%$ in the Ber group, and $0.81 \pm 0.141 \%$ in the Ber + antiCD25 group. The Ber group had a significantly higher percentage of these cells than the Derf $(p=0.001)$ or Ber + anti-CD25 groups $(p=0.023$; Figure $6 B)$.

\section{Discussion}

Anti-inflammatory effects of Ber have been reported in several autoimmune diseases. Ber has been reported to block the induction of edema on mouse ears by 12 -O-tetradecanoylphor-

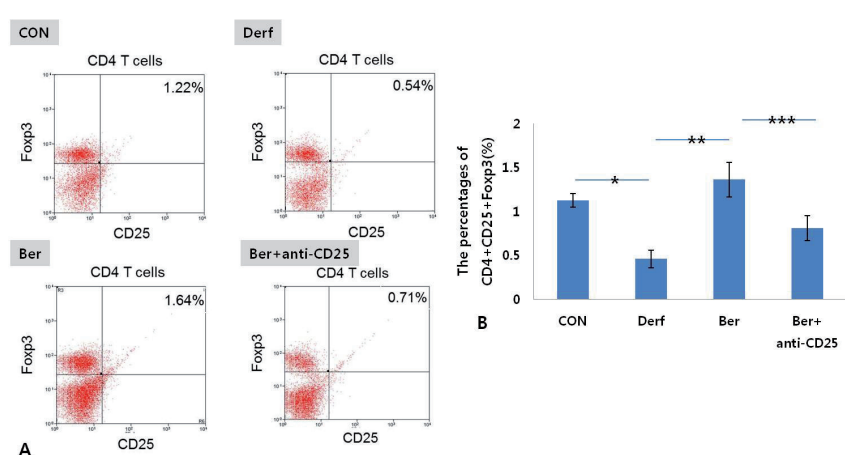

Figure 6. (A) Flow cytometric analysis of CD4+CD25+Foxp3+T-cell subsets. Representative fluorescence-activated cell sorting analysis in each group. RU, upper right quadrant, which represents CD4+CD25+Foxp3+ T cells. (B) The percentage of splenic mononuclear cells that were CD4+CD25+Foxp3+T cells. Error bars indicate standard deviations. * $\mathrm{p}<$ 0.05 , vs. the Derf group, ${ }^{* *} p<0.05$, vs. the control group.

bol-13-acetate and to inhibit cyclooxygenase-2 transcriptional activity, through regulation of activator protein 1 transcription factor ${ }^{(14)}$. In an earlier study, it was demonstrated that Ber could directly inhibit IL-17 production by $C D 4^{+} T$ cells and the dendritic cell-mediated differentiation of $C D 4^{+} I L-17^{+} T$ cells from Vogt-Koyanagi-Harada patients ${ }^{(15)}$. They showed that Ber significantly inhibited the TNF-a induced expression of IL-6, IL-8, and monocyte chemoattractant protein 1 in human retinal pigment epithelial-19 cells at both the protein and mRNA level, and that it downregulated phosphorylation of p38, extracellular signal regulated kinase $1 / 2$, and c-Jun $\mathrm{N}$-terminal kinase ${ }^{(15)}$. These data prompted investigation of the possibility of using Ber in the treatment of $A R$, the effect of which would be mediated by $\mathrm{CD}^{+} \mathrm{T}$ cells and various antigen-presenting cells. Several studies support Ber as an anti-atopic agent. Mast cells play an important role in the pathogenesis of $A R$, contributing in both early and late allergic responses. It has been shown that Ber can regulate allergic responses by inhibiting mast cells through inhibition of TSLP in an asthma model ${ }^{(7)}$.

In our study, repeated exposure to Derf induced a T helper 2 (Th2) milieu in mice, with a corresponding increase in symptom scores, serum IgE levels, GATA-3, IL-4, and IL-13 mRNA levels in the nasal mucosa, and tissue eosinophil counts. Ber treatment resulted in a significant reduction in serum IgE levels, GATA-3, IL-4, and IL-13 mRNA levels and tissue eosinophil counts in the Ber group compared with the Derf group. Thus, these results indicate that Ber suppresses the Th2 response.

We attempted to explore the pathway of the anti-allergic effects of Ber in an AR mouse model. One cellular mechanism for maintaining immune tolerance to foreign antigens, including allergens, is the natural Foxp3+ Treg cells population ${ }^{(16-18)}$. It 
has been shown, in allergy, that Treg cells can be transformed, conferring specific tolerance to subsequent challenges with the allergen ${ }^{(19,20)}$. In our study, Foxp3 mRNA levels were increased significantly in the Ber group compared with those in the Derf group. The percentage of $\mathrm{CD} 4^{+} \mathrm{CD} 25^{+} \mathrm{Foxp}^{+} \mathrm{T}$ cells, determined by flow cytometry, was also increased significantly in the Ber group compared with the Derf group. Cell-surface CD25 is a well-known marker of Tregs. It has been documented that antiCD25 mAb treatment reversed the effects of BCG/BCG-dendritic cells. In our study, in Ber + anti-CD25 group, Th2 cytokines were increased significantly, and IL-10 and Foxp3 mRNA levels were decreased significantly.

The immunosuppressive cytokine IL-10 has also been implicated in Treg cell function. As a short-range suppressive humoral factor involved in Treg-mediated suppression, IL-10 was first suspected to mediate Treg suppression ${ }^{(16,17)}$. In our study, we showed that IL-10 levels were increased significantly in the Ber group versus the Derf group. Ber showed a tendency to increase the number of $\mathrm{CD}^{+} \mathrm{CD} 25^{+}$Foxp $3^{+} \mathrm{T}$ cells. These findings suggest that Ber may have a novel property as an immunomodulator in controlling allergic inflammation.

\section{Conclusions}

In this study, we showed that Ber treatment inhibited allergic responses in a mouse model of AR. Additionally, anti-CD-25 $\mathrm{mAb}$ treatment largely reversed the effects of Ber. These results suggest that the inhibitory effect of Ber in our mouse model of AR is mediated by Tregs. Furthermore, this novel compound may provide a new strategy for treating or controlling AR.

\section{Acknowledgement}

The experiment was performed with the approval of the Institutional Animal Care and Use Committee at the Catholic University of Korea. Funded by Basic Science Research Program through the National Research Foundation of Korea (NRF) funded by the Ministry of Education, Science, and Technology (2011-0026915)

\section{Author contributions}

BYK: analysis and presentation; HRP: analysis; HGJ: analysis; SWK: design, conduct, analysis, and presentation.

\section{Conflict of interest}

None

\section{References}

1. Dykewicz MS, Fineman S, Skoner DP, et al. Diagnosis and management of rhinitis: complete guidelines of the Joint Task Force on Practice Parameters in Allergy, Asthma and Immunology. American Academy of Allergy, Asthma, and Immunology. Ann Allergy Asthma Immunol. 1998; 81: 478-518.

2. $\mathrm{Hu} Z$, Jiao Q, Ding J, et al. Berberine induces dendritic cell apoptosis and has therapeutic potential for rheumatoid arthritis. Arthritis Rheum. 2011; 63: 949-959.

3. Cui G, Qin X, Zhang Y, et al. Berberine differentially modulates the activities of ERK, p38 MAPK, and JNK to suppress Th17 and Th1 T cell differentiation in type 1 diabetic mice. $J$ Biol Chem. 2009: 284: 28420-28429.

4. Qin X, Guo BT, Wan B, et al. Regulation of Th1 and Th17 cell differentiation and amelioration of experimental autoimmune encephalomyelitis by natural product compound berberine. J Immunol. 2010; 185 1855-1863.

5. Ma X, Jiang Y, Wu A, et al. Berberine attenuates experimental autoimmune encephalomyelitis in C57 BL/6 mice. PLoS One. 2010; 5: e13489.

6. Yang Y1, Qi J, Wang Q, et al. Berberine suppresses Th17 and dendritic cell responses. Invest Ophthalmol Vis Sci. 2013; 54: 2516 2522

7. Moon PD, Choi IH, Kim HM. Berberine inhibits the production of thymic stromal lymphopoietin by the blockade of caspase-1/NF-kB pathway in mast cells.Int Immunopharmacol. 2011; 11: 1954-1959.

8. Zhou C, Kang XD, Chen Z. A synthetic Toll- like receptor 2 ligand decreases allergic immune responses in a mouse rhinitis model sensitized to mite allergen. Zhejiang Univ Sci B. 2008; 9: 279-285.

9. Wang W, Zhu Z, Zhu B, Ma Z. Peroxisome proliferator-activated receptor-gamma agonist induces regulatory $T$ cells in a murine model of allergic rhinitis. Otolaryngol Head Neck Surg. 2011; 144: 506-513.

10. Minty A, Chalon P, Deroca JM, et al. Interleukin-13 is a new human lymphokine regulating inflammatory and immune responses. Nature. 1993; 362: 248-250.

11. Szabo SJ, Kim ST, Costa GL, et al. A novel transcription factor, T-bet, directs Th1 lineage commitment. Cell. 2000; 100: 655-669.

12. Yamashita M, Ukai-Tadenuma M, Miyamoto T, et al. Essential role of GATA3 for the maintenance of type 2 helper T (Th2) cytokine production and chromatin remodeling at the Th2 cytokine gene loci. J Biol Chem 2004; 279: 26983-26990

13. Fields ML, Hondowicz BD, Metzgar MH, et al. CD4+ CD25+ regulatory $T$ cells inhibit the maturation but not the initiation of an autoantibody response. J Immunol. 2005; 175: 4255-4264

14. Yasukawa K, Takido M, Ikekawa $T$, et al. Relative inhibitory activity of berberine-type alkaloids against 12-O-tetradecanoylphorbol-13-acetateinduced inflammation in mice. Chem Pharm Bull. 1991; 39: 1462-1465.

15. Yang Y1, Qi J, Wang Q, Du L, et al. Berberine suppresses Th17 and dendritic cell responses. Invest Ophthalmol Vis Sci. 2013 5; 54: 2516-2522.
16. Rudensky AY. Regulatory T cells and Foxp3. Immunol Rev. 2011; 241: 260-268.

17. Sakaguchi S, Wing K, Miyara M. Regulatory T cells - a brief history and perspective. Eur J Immunol. 2007; 37: S116-123.

18. Valencia X, Lipsky PE. CD4+CD25+Foxp3+ regulatory $T$ cells in autoimmune diseases. Nat Clin Pract Rheumatol. 2007; 3: 619-626.

19. Cottrez F, Hurst SD, Coffman RL, et al. $T$ regulatory cells 1 inhibit a Th2-specific response in vivo. J Immunol. 2000; 165: 4848-4853.

20. Navarro S, Cossalter G, Chiavaroli C, et al. The oral administration of bacterial extracts prevents asthma via the recruitment of regulatory $T$ cells to the airways. Mucosal Immunol. 2011; 4: 53-65.

Soo Whan Kim, M.D., Ph.D. Department of Otolaryngology-Head and Neck Surgery College of Medicine The Catholic University of Korea 505 Banpo-dong, Seocho-gu Seoul 137-701

Korea

Tel: 82-2-2258-6214

Fax: 82-2-535-1354

E-mail: kshent@catholic.ac.kr 